Baumgart, D.C. 32

Bisschops, R. 46

Boškoski, I. 3

Cesaro, P. 3

Chahoud, F. 41

Costamagna, G. 3

Djuranovic, S. 27

Faiss, S. 60

Familiari, P. 3

Fry, L.C. 27

Goetz, M. 36

Gotthardt, D. 41

Hartmann, D. 17

Jovanovic, I. 27
Malfertheiner, P. 27

Mönkemüller, K. 27

Mössner, J. 9

Perri, V. 3

Pox, C. 56

Riemann, J.F. 53

Sauer, P. 41

Schreyer, A.G. 22

Schultz H.-J. 1

Stroszczynski, C. 22

Tringali, A. 3

Ugljesic, M. 27

Vormbrock, K. 27

Zimmermann, L. 27

\title{
Subject Index Vol. 29, Suppl. 1, 2011
}

Acute pancreatitis 9

Autofluorescence imaging 36

Biliary neoplasia 3

Cancer 32

Capsule endoscopy 17

- retention 17

Choledocholithiasis 3

Chromoendoscopy 36

Chronic pancreatitis 9

Colonoscopy 60

Colorectal cancer 36, 53, 60

- _ screening 56

Computed tomography 22

Confocal endomicroscopy 46

- laser endomicroscopy 36

Crohn's disease 17, 32, 36

Dominant stricture 41

Double-balloon enteroscopy 27

Dysplasia-associated lesion or mass 46

Early detection 3

Endoscopic retrograde cholangiography 41

- treatment 41

Endoscopy 53

- screening 32

Enteroscopy 27

Exocrine pancreatic physiology 9
Fecal occult blood testing 56

Genetic stool testing 56

Genetics 9

High definition 46

High-definition endoscopy 36

Inflammatory bowel disease 32

Interval cancer 60

Intraepithelial neoplasia 36

M2-PK 56

Magnetic resonance cholangiography 41

- - imaging 22

Missed cancer 60

Mucosal healing 17

Narrow band imaging 36

Obscure gastrointestinal bleeding 27

Palliation 3

Polyps 46

Sclerosing cholangitis 41

Screening colonoscopy 60

- programs, Europe 53

Small bowel 22

- - diagnosis 22

- - polyps 27

Stool tests 56

Therapeutic enteroscopy 27

Ulcerative colitis 32,36 